\title{
Cisplatin-Based Chemotherapy Eligibility: Discrepancy Between Serum Creatinine Level and Glomerular Filtration Rate
}

\author{
Kambiz Novin, ${ }^{1}$ Shiva Moghadam,, Nafiseh Mortazavi, ${ }^{1,}$ Farnaz Hosseini Kamal, ${ }^{3}$ \\ Helaleh Khoshbakht Ahmadi, ${ }^{1}$ and Ahamd Ameri ${ }^{1}$ \\ ${ }^{1}$ Clinical Oncology Department, Shahid Beheshti University of Medical Sciences, Tehran, IR Iran \\ ${ }^{2}$ Pathology Department, Shahid Beheshti University of Medical Sciences, Tehran, IR Iran \\ ${ }^{3}$ Department of Chemical and Biological Engineering, Chalmers University of Technology, Gothenburg, Sweden \\ *Corresponding author: Shiva Moghadam, Clinical Oncology Department, Shahid Beheshti University of Medical Sciences, Tehran, IR Iran. Tel: +98-2173432621, Fax: \\ +98-2177552056, E-mail: moghadamshiva@yahoo.com \\ Received: May 1, 2015; Accepted: May 15, 2015
}

\begin{abstract}
Background: Serum creatinine level is frequently used as a measure for renal function assessment. However, there are some situations in which patients may suffer significant renal impairment but serum creatinine levels remain within normal ranges.

Objectives: We conducted this study to evaluate the discrepancy between serum creatinine (SCr) level and glomerular filtration rate (GFR) in determining the eligibility for cisplatin-based chemotherapy among cancer patients.

Patients and Methods: A total of 198 cancer patients had received cisplatin-based chemotherapy at Jorjani Cancer Center, Emam Hossein Hospital, Tehran, Iran were retrospectively investigated. The discordance between SCr level and calculated GFR by Cockcroft-Gault equation was analyzed.

Results: 130 patients (66\%) were men and 68 (34\%) were women with mean age of 54.5 years. Squamous cell carcinoma (SCC) and head and neck were the most common primary tumor histology and site respectively. Of 165 patients with available data to calculate eGFR, 45 (27.3\%) had normal kidney function based solely on SCr levels, but their GFR was less than $60 \mathrm{~mL} / \mathrm{min}$ (renal dysfunction). The discordance between SCr and GC calculated GFR values were most pronounced in the older age, transitional cell carcinoma histology and bladder primary site. Conclusions: This study shows that SCr level alone may not be a reliable measure of normal kidney function to determine eligibility for cisplatine-based chemotherapy.
\end{abstract}

Keywords: Serum; Creatinine; Glomerular Filtration Rate; Cisplatin; Cancer

\section{Background}

Renal function assessment is critical in determining the eligibility or need to dose modification of nephrotoxic drugs in clinical practice (1). Measuring glomerular filtration rate (GFR) is a widely accepted index of renal function. Although the most common method of GFR assessment is urine collection for evaluation of creatinine clearance, most of the time this test is inconvenient for the patient and also may overestimate GFR due to renal tubular secretion of creatinine (2). So, attempts have been focused on formulas that estimate GFR without collecting patient's urine (3). In this respect the most common formulas used by laboratories to predict GFR are Cockcroft and Gault (CG) (4) and modification of diet in renal disease (MDRD) (5) equations that provide rapid methods of calculating GFR through serum creatinine (SCr).

Currently, in clinical practice and specifically oncology clinics many use serum creatinine level as a measure for renal function assessment. However, there are some situations in which patients may suffer significant renal impairment though serum creatinine levels remain within normal ranges $(1,6)$. Despite discrepancies between serum creatinine and GFR to show normal kidney status, many oncologists continue to interpret a normal SCr level as an indicator of a normal renal function. This can be more dangerous when patients are candidated for systemic chemotherapy regimens containing nephrotoxic drugs like cisplatin $(1,7)$.

\section{Objectives}

The present study was designated to magnitude the misinterpretation of renal function based solely on serum creatinine levels among cancer patients received cisplatin containing chemotherapy.

Copyright ( 2015, Iranian Society of Clinical Oncology. This is an open-access article distributed under the terms of the Creative Commons Attribution-NonCommercial 4.0 International License (http://creativecommons.org/licenses/by-nc/4.0/) which permits copy and redistribute the material just in noncommercial usages, provided the original work is properly cited. 


\section{Patients and Methods}

This is a retrospective study conducted at Jorjani cancer center, Emam Hossein hospital, Tehran, Iran. A total of 198 cancer patients had received cisplatin-based chemotherapy were investigated. With a previously prepared fact sheet the following data for the patients were gathered from their medical records: patient's age, sex, weight and height, primary tumor site, primary tumor histology, kind of chemotherapy regimen administered and patient's serum creatinine level before starting the chemotherapy. Serum creatinine measurements were done based on Jaffe reaction (alkaline picrate method) (8). For each patient, GFR was estimated by Cockcroft and Gault equation as follows (Equation 1):

(1) $\mathrm{eC}_{\mathrm{cr}}=\frac{(140 \times \text { Age }) \times \text { Mass (in kilograms }) \times[0.85 \text { if Female }]}{72 \times \text { Serum Creatinine }\left(\text { in } \frac{\mathrm{mg}}{\mathrm{dL}}\right)}$

Upper limit of normal for serum creatinine level was defined as $1.4 \mathrm{mg} / \mathrm{dL}$ for males and $1.3 \mathrm{mg} / \mathrm{dL}$ for females (9). Estimated GFR (eGFR) was classified as: normal (eGFR $\geq 60 \mathrm{~mL} / \mathrm{min}$ and eligible for full dose cisplatin); borderline $(45 \leq \mathrm{eGFR}<60 \mathrm{~mL} / \mathrm{min}$ and eligible for modified dose cisplatin) and low (eGFR $<45 \mathrm{~mL} / \mathrm{min}$ and ineligible for cisplatin) (10). The discrepancy between serum creatinine level and eGFR in determining the eligibility for cisplatin-based chemotherapy was analyzed. This study was approved by the local scientific and ethical committee.

\subsection{Statistical Analysis}

To evaluate the relation of different factors with the eGFR level, Spearman correlation, Mann-Whitney and Kruskall-Wallis tests were used. Roc curve analysis with Youden index was applied to extract the best cutoff of Creatinine. In the last step diagnostic values such as the sensitivity, specificity and negative and positive values were demonstrated for these cutoffs. All statistical analysis performed by SPSS (Version 21.0, IBM Co. Chicago, IL). A $P$ value of $<0.05$ was considered significant.

\section{Results}

\subsection{Patient and Tumor Characteristics}

Of 198 included patients, 130 (66\%) were men and 68 (34\%) were women with mean age of 54.5 years (16 to 83 years) and mean weight of $68 \mathrm{~kg}$ (39 to $175 \mathrm{~kg}$ ). Squamous cell carcinoma (SCC) and head and neck region were the most common primary tumor histology and site respectively ( $86 \%$ and $67 \%$ of all patients respectively). Mean eGFR calculated by CG formula for all patients was $80 \pm 32 \mathrm{~mL} / \mathrm{min}$. Mean SCr level for all patients was $0.99 \mathrm{mg} / \mathrm{dL}$ (0.6 to 2.9 with median value of $1.00 \mathrm{mg} / \mathrm{dL}$ ). Median values for age, weight and eGFR were 56 years, $66 \mathrm{~kg}$ and $73 \mathrm{~mL} / \mathrm{min}$, respectively. Chemotherapy regimens administered to the patients were: weekly cisplatin; PF (cispaltin plus 5-fluorouracil); TPF (docetaxel plus cispaltin plus 5-fluorouracil) and other combinations with cisplatin. The study population characteristics are detailed in Table 1.

Table 1. The Study Population Characteristics ${ }^{a}$

\begin{tabular}{lc}
\hline Variable & Values $^{\mathrm{b}}$ \\
\hline Sex & \\
Male & $130(66)$ \\
Female & $68(34)$ \\
Age, $\mathbf{y}$ & \\
$\leq 30$ & $12(6)$ \\
$31-60$ & $116(59)$ \\
$>60$ & $70(35)$
\end{tabular}

\section{Weight, kg}

$\leq 50 \quad 20(10)$

$51-80 \quad 135(68)$

$>80 \quad 29(15)$

missing $14(7)$

BSA, $\mathbf{m}^{2}$

$\begin{array}{lc}\leq 1.40 & 15(8) \\ 1.41-1.80 & 135(68) \\ >1.80 & 38(19) \\ \text { Missing } & 10(5)\end{array}$

Primary Tumor Site

$\begin{array}{lc}\text { Head and Neck } & 132(67) \\ \text { Cervix } & 46(23) \\ \text { Bladder } & 18(9) \\ \text { Esophagus } & 1(0.5) \\ \text { Missing } & 1(0.5)\end{array}$

\begin{tabular}{lc} 
Primary Tumor Histology & \\
\hline SCC & $170(86)$ \\
\hline TCC & $19(9.5)$ \\
\hline Adenocarcinoma & $8(4)$ \\
Other & $1(0.5)$
\end{tabular}

Chemotherapy Regimen

\begin{tabular}{lc} 
Weekly cisplatin & $148(75)$ \\
PF & $35(10)$ \\
TPF & $46(18)$ \\
Cisplatin plus other agents & $20(23)$ \\
\hline
\end{tabular}

a Abbreviations: BSA, body surface area; $\mathrm{PF}$, cisplatin plus 5-fluorouracil; SCC, squamous cell carcinoma; TCC, transitional cell carcinoma; TPF, docetaxel plus cisplatin plus 5-fluorouracil.

b Data are presented as No. (\%). 


\subsection{SCr and eGFR Analysis}

One hundred twelve patients (68\% of all) had normal kidney function (eGFR $\geq 60 \mathrm{~mL} / \mathrm{min}$ ) before starting chemotherapy. In 11 patients (about 7\%) eGFRs were below the acceptable level for cisplatin administration. Others had eGFRs indicating borderline renal functions. Table 2 shows the correlation between eGFR and different characteristics of patients' population. As shown in this table, eGFR tended to be higher in younger ages $(P<0.001)$, higher body weights and higher body surface areas $(\mathrm{P}=0.001$ and $\mathrm{P}<0.001$, respectively). Considering primary tumor histology and site, low eGFRs ( $<45 \mathrm{~mL} / \mathrm{min}$ ) were observed more often in transitional cell carcinoma (TCC) and bladder subgroup, respectively $(\mathrm{P}<0.001)$.

Table 2. The Correlation Between eGFR and Patient Characteristics a,b

\begin{tabular}{|c|c|c|c|c|}
\hline \multirow[t]{2}{*}{ Parameter } & \multicolumn{3}{|c|}{ eGFR, mL/min } & \multirow[t]{2}{*}{ PValue } \\
\hline & $<45$ & $46-59$ & $\geq 60$ & \\
\hline Total & $11(6.7)$ & $41(25.0)$ & $112(68.3)$ & - \\
\hline Age, $y$ & & & & $<0.001$ \\
\hline$\leq 30$ & $0(0.0)$ & $0(0.0)$ & $9(100.0)$ & \\
\hline $31-60$ & $4(4.1)$ & $14(14.4)$ & $79(81.4)$ & \\
\hline$>60$ & $7(11.9)$ & $27(45.8)$ & $25(42.4)$ & \\
\hline Sex & & & & 0.715 \\
\hline Male & $6(5.5)$ & $28(25.5)$ & $76(69.1)$ & \\
\hline Female & $5(9.1)$ & $13(23.6)$ & $37(67.3)$ & \\
\hline Weight, kg & & & & $<0.001$ \\
\hline$\leq 50$ & $4(22.2)$ & $8(44.4)$ & $6(33.3)$ & \\
\hline $51-80$ & $6(5.0)$ & $32(26.9)$ & $81(68.1)$ & \\
\hline$>80$ & $1(3.6)$ & $1(3.6)$ & $26(92.9)$ & \\
\hline BSA, $\mathbf{m}^{2}$ & & & & 0.001 \\
\hline$\leq 1.40$ & $2(15.4)$ & $7(53.8)$ & $4(30.8)$ & \\
\hline $1.41-1.80$ & $8(6.8)$ & $30(25.6)$ & $79(67.5)$ & \\
\hline$>1.80$ & $1(2.9)$ & $4(11.8)$ & $29(85.3)$ & \\
\hline Primary Tumor Histology & & & & $<0.001$ \\
\hline SCC & $6(4.3)$ & $30(21.6)$ & $103(74.1)$ & \\
\hline TCC & $5(27.8)$ & $8(44.4)$ & $5(27.8)$ & \\
\hline Adenocarcinoma & $0(0.0)$ & $3(42.9)$ & $4(57.1)$ & \\
\hline Other & $0(0.0)$ & $0(0.0)$ & $1(100.0)$ & \\
\hline Primary Tumor Site & & & & $<0.001$ \\
\hline Cervix & $2(5.1)$ & $11(28.2)$ & $26(66.7)$ & \\
\hline Esophagus & $0(0.0)$ & $0(0.0)$ & $1(100.0)$ & \\
\hline Head and neck & $4(3.7)$ & $22(20.6)$ & $81(75.7)$ & \\
\hline Bladder & $5(29.4)$ & $8(47.1)$ & $4(23.5)$ & \\
\hline
\end{tabular}

a Data are presented as No. (\%).

b Abbreviations: BSA, body surface area; eGFR, estimated glomerular filtration rate; SCC, squamous cell carcinoma; TCC, transitional cell carcinoma. 
Of 165 patients with available data to calculate eGFR, 52 (31.5\%) had abnormal GFR calculated by Cockcroft and Gault equation, while 7 (4.2\%) had overtly abnormal renal function with Scr values. Interestingly, 45 (27.3\%) patients had normal kidney function based solely on SCr levels, but their GFR was less than $60 \mathrm{~mL} / \mathrm{min}$ (renal dysfunction). The discordance between SCr and CG calculated GFR values were most pronounced in the older age, transitional cell carcinoma histology and bladder primary site (Tables 3 and 4 ).

Another notable result of the present study was the inability of SCr level to detect patients with severe renal disfunction. Eleven patients in our study had eGFRs less than $45 \mathrm{ml} / \mathrm{min}$ in whom cisplatin could not be administered but only three of them (27\%) was detectable by considering the abnormal serum creatinine levels (Table 3).

According to the ROC curve, the best cut-off value for SCr was 0.98. SCr values less than $0.98 \mathrm{mg} / \mathrm{dl}$ with a sensitivity of $60 \%$, specificity of $83 \%$ and positive predictive value of $88 \%$ could predict a normal renal function (eGFR $\geq 60 \mathrm{~mL} / \mathrm{min}$ ) (Figure 1).

\begin{tabular}{|c|c|c|c|c|c|}
\hline \multirow[t]{2}{*}{ Serum Creatinine Level } & \multicolumn{4}{|c|}{ eGFR, $\mathrm{mL} / \mathrm{min}$} & \multirow[t]{2}{*}{ P Value } \\
\hline & GFR $<45$ & $45 \leq$ GFR $<60$ & GFR $\geq 60$ & Total & \\
\hline Normal & $8(4.8)$ & $38(23.0)$ & $112(67.9)$ & $158(95.8)$ & 0.000 \\
\hline Abnormal & $3(1.8)$ & $3(1.8)$ & $1(0.6)$ & $7(4.2)$ & \\
\hline Total & $11(6.7)$ & $41(24.8)$ & $113(68.5)$ & $165(100.0)$ & \\
\hline
\end{tabular}

a Data are presented as No. (\%).

b Abbreviation: eGFR, estimated glomerular filtration rate.

Table 4. Prevalence of Abnormal eGFR and SCr Values by Patient and Tumor Characteristics ${ }^{\text {a,b }}$

\begin{tabular}{lcccc}
\hline & Total & eGFR $<\mathbf{6 0} \mathbf{~ m L} / \mathbf{m i n}$ & Abnormal SCr & Difference \\
\hline All Patients & $165(100)$ & $52(31.5)$ & $7(4.2)$ & $45(27.3)$ \\
\hline Sex & & & $5(4.5)$ & $29(26.4)$ \\
\hline Male & $110(66.7)$ & $34(30.9)$ & $2(3.6)$ & $16(29.1)$ \\
\hline Female & $55(33.3)$ & $18(32.7)$ & & $0(0)$ \\
Age, $\mathbf{y}$ & & & $0(0)$ & $14(14.5)$ \\
$\leq 30$ & $9(5.4)$ & $0(0)$ & $4(4.1)$ & $31(53)$
\end{tabular}

Primary Tumor Histology

\begin{tabular}{lcccc|}
\hline TCC & $18(10.9)$ & $13(72.2)$ & $4(22.2)$ & $9(50)$ \\
\hline Non TCC & $147(89.1)$ & $39(26.5)$ & $3(2)$ & $36(24.5)$ \\
\hline Primary Tumor Site & & & $4(23.5)$ & $9(53)$ \\
\hline Bladder & $17(10.4)$ & $13(76.5)$ & $3(2)$ & $36(24.5)$ \\
\hline Non bladder & $147(89.6)$ & $39(26.5)$ & & \\
\hline
\end{tabular}

a Data are presented as No. (\%).

b Abbreviations: eGFR, estimated glomerular filtration rate; TCC, transitional cell carcinoma. 


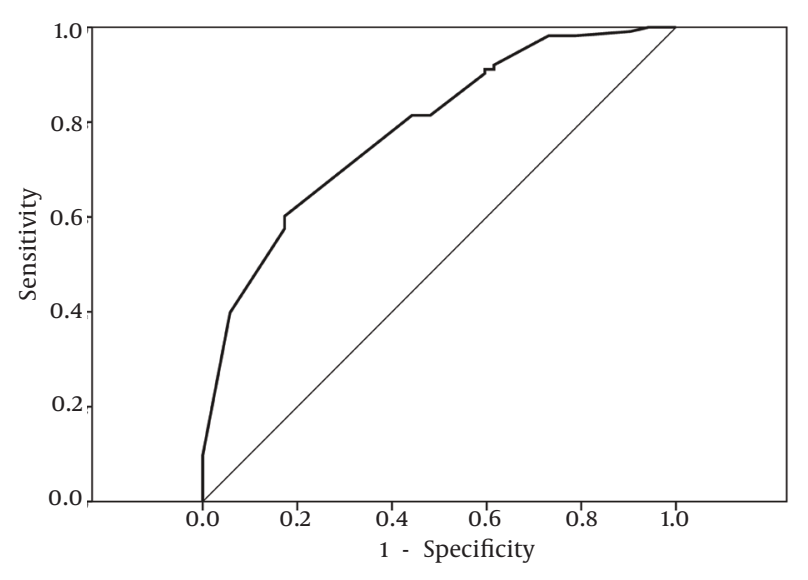

Figure 1. Receiver Operating Characteristic (ROC) Curve for Serum Creatinine Levels Predicting Normal Renal Functions (eGFRs $\geq 60 \mathrm{~mL} / \mathrm{min}$ )

\section{Discussion}

A main oncologists' concern in prescribing cisplatin containing regimens has been the probable renal impairment caused by this drug. A recent meta-analysis on clinical trials using serum creatinine versus calculated glomerular filtration rate as indicators of renal sufficiancy for chemotherapy prescription showed that cisplatinassociated nephrotoxicity might be higher when SCr was used instead of GFR as eligibility criteria (11). On this purpose some guidelines for cisplatin dose calculation and dose adjustment using the baseline renal function of patients have been developed. On the other hand, in many circumstances we see that oncologists prescribe cisplatin only if the patients have got a normal serum creatinine without considering their creatinine clearance. This study reveals the discordance between these two methods of renal sufficiency prediction.

According to our results, there were a significant relationship between the patients' eGFR and some variables including age, weight, tumor histology and primary site. By increasing age, patients were less likely to have a normal eGFR. All of the patients aged 30 years or younger had normal eGFRs whereas only in $42 \%$ of patients over 60 years old eGFRs were normal $(\mathrm{P}<0.001)$. Normal eGFR was also significantly more common in higher weight groups. According to this study the rate of normal eGFR was 93\% in the patients over $80 \mathrm{kgs}$ versus $33 \%$ in the patients weighing up to $50 \mathrm{kgs}(\mathrm{P}<0.001)$. These differences in eGFRs between the age and weight groups could be explained by the CG equation used for estimating GFR in this study. There wasn't any significant difference in estimated renal function between men and women in the present study that seemed rational due to considering sex coefficient in calculating GFR by the CG equation. An interesting finding of this study was the significant effect of tumor histology and site on estimated GFR. About $29 \%$ of patients with transitional cell carcinoma (TCC) had eGFRs less than $45 \mathrm{~mL} / \mathrm{min}$ so, they were not eligible for cisplatin administeration, while such low rates of eGFRs were observed in $4 \%$ and $0 \%$ of patients with squamous cell carcinoma (SCC) and adenocarcinoma respectively $(\mathrm{P}<0.001)$. There was also a similar difference in eGFRs with respect to the primary tumor site. All bladder cancers in this study were of TCC histology so this could be the reason for above findings with respect to the tumor sites and histologic subtypes. Although defining the exact factor associated with this proximity seems difficult.

Our study suggests that serum creatinine level may not be a reliable indicator of renal sufficiency in cancer patients candidated for cisplatin-based chemotherapy. This result is in concordance with an Indian study. Kannapiran et al in their study on 928 out patients showed that 270 patients $(29.1 \%)$ had renal dysfunction on the basis of eGFR $\left(60 \mathrm{~mL} / \mathrm{min} / 1.73 \mathrm{~m}^{2}\right)$. However, with SCr only 162 (17.5\%) patients had abnormal renal function and SCr values misrepresented 108 (11.6\%) patients with impaired kidney function. This discrepancy was even more pronounced either among female or older patients (1). A probable explanation for lower discrepancy between $\mathrm{SCr}$ and eGFR in their study (11.6\% versus $27.3 \%$ in the present study) is using MDRD equation for calculating the patients' GFRs by them. Another reason may be different populations of these two sudies, as the patients in our study were known cases of cancer but Kannapiran et al. conducted the study on non-cancer patients.

A practical result of the present study was detection of a cut off value for serum creatinine level that could predict whether the patients were allowed to take complete dose of cisplatin based on their eGFR or not. For all the patients this cut off was 0.98 which meant serum creatinine levels bellow $0.98 \mathrm{mg} / \mathrm{dL}$ predicted normal renal functions (eGFRs $\geq 60 \mathrm{~mL} / \mathrm{min}$ ) in $88 \%$ of cases.

Using only SCr level may not be a reliable index of normal renal function in considering patients for cisplatine based chemotherapy and more cautions should be done in this respect.

\section{References}

1. Kannapiran M, Nisha D, Madhusudhana Rao A. Underestimation of impaired kidney function with serum creatinine. Indian J Clin Biochem. 2010;25(4):380-4.

2. Diamandopoulos A, Goudas P, Arvanitis A. Comparison of estimated creatinine clearance among five formulae (CockroftGault, Jelliffe, Sanaka, simplified 4-variable MDRD and DAF) and the 24hours-urine-collection creatinine clearance. Hippokratia. 2010;14(2):98-104.

3. Rahn KH, Heidenreich S, Bruckner D. How to assess glomerular function and damage in humans. J Hypertens. 1999;17(3):309-17.

4. Cockcroft DW, Gault MH. Prediction of creatinine clearance from serum creatinine. Nephron. 1976;16(1):31-41.

5. Levey AS, Coresh J, Greene T, Stevens LA, Zhang YL, Hendriksen S, et al. Using standardized serum creatinine values in the modification of diet in renal disease study equation for estimating glomerular filtration rate. Ann Intern Med. 2006;145(4):247-54.

6. Levey AS, Perrone RD, Madias NE. Serum creatinine and renal function. Annu Rev Med. 1988;39:465-90.

7. Stevens LA, Coresh J, Greene T, Levey AS. Assessing kidney func- 
tion--measured and estimated glomerular filtration rate. $N$ Engl J Med. 2006;354(23):2473-83.

8. Weber JA, van Zanten AP. Interferences in current methods for measurements of creatinine. Clin Chem. 1991;37(5):695-700.

9. Oh MS. Evaluation of renal function, water, electrolytes and acid-base balance. In: McPherson RA, Pincus MR, editors. HENRY'S Clinical Diagnosis and Management by Laboratory Methods. 22th ed. Philadelphia: Saunders; 2011. pp. 169-92.
10. Provincial Health Services Authority. BCCA Protocol Summary for Treatment of Locally Advanced Squamous Cell Carcinoma of the Head and Neck with Docetaxel,cisplatin and Infusional Fluorouracil. Available from: http://www.bccancer.bc.ca/.

11. Dahal A, Bellows BK, Sonpavde G, Galsky MD, Agarwal N. Cisplatin-associated nephrotoxicity in clinical trials using serum creatinine $(\mathrm{SCr})$ versus calculated glomerular filtration rate (GFR) as inclusion criterion: A meta-analysis. J Clin. 2013;31(6) 